\title{
Factores moduladores de la respuesta agresiva tras la exposición a videojuegos violentos
}

\author{
Alejandro de la Torre-Luque ${ }^{1 *}$ y Luis Valero-Aguayo ${ }^{2}$
}

${ }^{1}$ Universidad de Granada, ${ }^{2}$ Universidad de Málaga

\begin{abstract}
Resumen: En ciertas situaciones se ha asociado la influencia de los videojuegos violentos con las conductas agresivas y/o delictivas. El presente estudio pretende destacar un efecto de la exposición a un videojuego violento de coches sobre la elicitación a corto plazo, en las respuestas de agresión e ira tras la exposición. Para ello, 47 adolescentes fueron expuestos al videojuego violento y a uno no violento durante 30 minutos. Se midieron ansiedad e ira autoinformadas pre-postest, así como la ejecución en una tarea indirecta de agresión. Se halló un efecto principal de la edad y de interacción de ésta con el tipo de videojuego y el sexo. Asimismo, se halló un efecto pre-postest en ira estado tras la exposición al videojuego violento. Por otro lado, se encontró un modelo predictor para la ejecución de la respuesta agresiva tras la presentación del videojuego violento con la edad e ira preexposición como factores. En conclusión, se destaca un efecto de priming de la ira a corto plazo y la edad tras la exposición al videojuego violento, en la respuesta agresiva. La presencia de moduladores de los efectos de contenidos violentos puede esclarecer su naturaleza y ayudar a predecir conductas delictivas.

Palabras clave: Ira; agresión indirecta; videojuegos violentos; adolescentes; modelo general de la agresión; edad; priming.
\end{abstract}

\section{Introducción}

En marzo del año 2000 la prensa española publicaba el suceso ocurrido en la provincia de Murcia, en el que un adolescente asesinaba a varios miembros directos de su familia utilizando como arma una catana (Agencia EFE, 2001). En algunos medios de comunicación se insinuó que los actos del adolescente emulaban la acción de un personaje del videojuego Final Fantasy VIII ${ }^{\circledR}$, en el que predominan escenas de violencia explícita y lenguaje con contenido obsceno (Entertainment Software Association, ESA, 2012). Asimismo, se desprendía la hipótesis de la relación entre la reiterada exposición a videojuegos y aprendizaje de las conductas violentas ejecutadas por los avatares de los mismos (Fernández y Noblejas, 2007).

En este sentido cabe destacar que en la literatura científica se encuentran evidencias de la amplia frecuencia de uso de videojuegos en población infantojuvenil (Chóliz y Marco, 2011; Gentile, 2009; Gentile y Walsh, 2002; Hallal, Bertoldi, Gonçalves y Victora, 2006; Huesmann y Taylor, 2006; Olds et al., 2009). Más concretamente, en España diversos estudios han indicado que los adolescentes se exponen entre $1 \mathrm{y}$ 3 horas al día a videojuegos (Bercedo et al., 2005; Wittmann, Arce y Santisteban, 2008).

Además, la elevada frecuencia de exposición de niños y adolescentes a los videojuegos se ha asociado en algunos estudios a bajo rendimiento escolar, sedentarismo, trastornos

\section{* Dirección para correspondencia [Correspondence address]:}

Alejandro de la Torre Luque Departamento de Personalidad, Evaluación y Tratamiento Psicológico. Facultad de Psicología. Universidad de Granada. Campus de Cartuja, s/n. 18011, Granada (España). E-mail: psicoatl@ugr.es
Title: Modulating factors of the aggressive response after the exposure to violent video games.

Abstract: The influence of violent video games has been associated with aggressive and/or criminal behaviours in several situations. The present research pretends to emphasize an effect of the exposure to a violent car video game on the activation of aggressive response and post-exposure anger levels. For this, a sample of 47 adolescent people was selected and exposed to a violent video game and a non violent one during 30 minutes. Selfreported anxiety and anger levels were measured before and after the video game exposures, moreover the performance in an indirect task of aggression. A main effect of the age and interaction of this with sex, and the kind of video game were found in the aggressive task. Likewise, a pre-postest effect on state anger levels was observed. On the other hand, a predictor model of the aggressive response elicitation was found with the age and pre-exposure anger as factors. In conclusion, it is outstanding the short term priming effect of violent video games on the aggressive behaviour after violent video game exposure. The existence of modulating factors on the effects of violent contents could clarify their features and help to predict criminal behaviours.

Key words: Anger; indirect aggression; violent video games; adolescent people; general aggression model; age; priming.

adictivos y otros problemas de conducta (Barlett, Anderson y Swing, 2009; Hallal et al., 2006; Holtz y Appel, 2011; Olson et al., 2007; Sharif y Sargent, 2006; Skoric, Teo y Neo, 2009). Además, se han encontrado pruebas empíricas a favor de la influencia de la exposición a videojuegos violentos en la respuesta agresiva humana. Se han señalado el aumento de creencias, estados afectivos y comportamientos propios de la agresión, así como el incremento de la activación fisiológica y el decremento de la conducta altruista (Adachi y Willoughby, 2011; Anderson, 2004; Anderson y Carnagey, 2009; Anderson el al., 2010; Baldaro et al., 2004; Bartholow, Sestir y Davis, 2005; Bösche, 2010; Fischer, Greitemeyer, Kastenmüller, Vogrincic y Sauer, 2011; Fleming y Rickwood, 2001; Wei, 2007).

Dichos efectos parecen más pronunciados en la adolescencia, principalmente en hombres (Anderson, 2004; Kirsh, 2003; Loeber y Stouthamer-Loeber, 1998); también se han mostrado consistentes bajo diferentes tipos de videojuegos violentos, como videojuegos de rol (Anderson et al., 2010; Bösche, 2010; Giumetti y Markey, 2007), de combate (Bluemke, Friedrich y Zumbach, 2010; Ferguson y Garza, 2011; Panee y Ballard, 2002; Wei, 2007), deportivos y de coches (Anderson y Carnagey, 2009; Fischer, Kastenmüller y Greitermeyer, 2010; Fischer, Kubitzki, Guter y Frey, 2007). También se ha observado una mayor presencia de ejecución de conductas arriesgadas tras la exposición a videojuegos de tipo competitivo (Fischer et al., 2011).

Se plantea el modelo general de la agresión (General $A g$ gression Model, GAM) como marco teórico para examinar el efecto de la exposición a videojuegos violentos en la elicitación de respuestas agresivas (Adachi y Willoughby, 2011; Anderson y Bushman, 2002; Barlett et al., 2009; Bushman y 
Huesmann, 2006; Huesmann, 2007). En el modelo se postula que la exposición a videojuegos violentos puede producir una facilitación de la agresión conductual (Berkowitz, 1984). Después de la exposición, se mantiene la activación y la tendencia a responder de forma agresiva transitoriamente, gracias al proceso de priming (Fischer, Guter y Frey, 2008; Giumetti y Markey, 2007; Huesmann, 2007; Huesmann y Taylor, 2006; Hummer et al., 2010).

Desde la literatura científica se extraen diversas evidencias empíricas señalando el efecto del uso de videojuegos sobre la respuesta agresiva: Anderson y otros (Anderson, 2004; Anderson y Dill, 2000; Anderson et al., 2010; Bushman y Huesmann, 2006; Huesmann, 2007) destacaron un efecto débil o moderado de la exposición a videojuegos violentos sobre la respuesta agresiva (en promedio, $.26<r<$ $.38)$.

Los autores indicaron que dicha potencia explicativa se debía principalmente a limitaciones metodológicas de muchos de estos estudios. No obstante, también hay que tener en cuenta el potencial peso de las variables moduladoras entre la exposición a videojuegos y la agresión, tales como impulsividad (Wittmann et al., 2008), ira (Giumetti y Markey, 2007; Jansz, 2005) y hostilidad (Baldaro et al., 2004; Barlett et al., 2007; Bartholow et al., 2005; Slater, Henry, Swaim y Anderson, 2003).

En el GAM se postula la ira como un facilitador de la respuesta agresiva tras la exposición a videojuegos violentos (Anderson y Bushman, 2002; Huesmann, 2007; Kerr y Schneider, 2008). Los resultados no son totalmente concluyentes en este respecto, quizá por las deficiencias de algunos estudios. Los estudios psicofisiológicos y de neuroimagen tampoco han sido mucho más esclarecedores (Arriaga, Esteves, Carneiro y Monteiro, 2006; Higuchi, Motohashi, Liu y Maeda, 2005; Hummer et al., 2010; Mathiak y Weber, 2006; Salminen y Ravaja, 2008).

Dada la posible influencia de la exposición a videojuegos de contenido violento sobre la elicitación de respuestas agresivas, esta investigación persigue señalar el efecto de la exposición a un juego violento de coches en adolescentes sobre la respuesta agresiva humana, evaluada con una prueba indirecta de agresión, así como el efecto de dicha exposición en los niveles de ansiedad e ira postests. Por otro lado, se pretende destacar la influencia de variables predictoras como la ira, la ansiedad, el sexo y la edad.

Se plantea que cuando los participantes se expongan a un videojuego violento de conducción mostrarán unos niveles superiores de respuestas en la tarea indirecta de agresión así como los niveles mayores postest en la ira. Por otro lado, se hipotetiza que se observarán diferencias con respecto al género y a la edad, en la respuesta agresiva en función de la exposición a un tipo de videojuego u otro. Por último, se plantea que se encontrará un modelo explicativo para las respuestas agresivas tras la exposición a los dos tipos de videojuegos en la que influya la ira estado y otras variables como el sexo, el género y la ira rasgo.

\section{Método}

\section{Participantes}

Se seleccionaron un total de 47 participantes, 29 varones y 18 mujeres, con un rango de edad de entre 12 y 15 años $(M$ = 13.06; $D T=1.06)$. Todos los participantes eran de raza blanca caucásica y residentes de la ciudad de Montilla, en la provincia de Córdoba (España); el total de los participantes estudiaba en dos institutos de dicha localidad en los cursos de primero y segundo de educación secundaria obligatoria. Los padres dieron su consentimiento escrito para dicha participación. Ninguno de los participantes disponía de licencia de ciclomotor o de coche.

\section{Instrumental}

Se usó el Inventario de Expresión de la Ira Estado-Rasgo para Niños y Adolescentes, STAXI-NA (del Barrio, Aluja y Spielberger, 2004; versión de del Barrio, Aluja y Spielberger, 2005). Se trata de un inventario que evalúa dos constructos que parten de la ira, estado y rasgo, en población infantil y adolescente. El instrumento está compuesto por 32 ítems con una escala tipo Likert con tres alternativas de respuesta. Las puntuaciones del inventario se dividen en tres factores principales, que a su vez cuentan con varios subfactores: ira estado (subfactores: sentimiento y expresión física y verbal), ira rasgo (subfactores: temperamento y reacción) y expresión de la ira (subfactores: expresión interna de la ira, expresión externa de la ira y control de la ira). Las propiedades psicométricas para muestra española (del Barrio et al., 2004) arrojan índices adecuados de fiabilidad (.53> $\alpha>.81$, para los cuatro factores) y validez (se observan correlaciones significativas con otros constructos tales como agresividad física y verbal).

También se empleó el Inventario de Ansiedad EstadoRasgo para Niños, STAIC (Spielberger, 1973; versión de Seisdedos, 1990). El instrumento se presenta como un inventario que mide ansiedad en niños de 8-15 años. Consta de 40 ítems con un formato de respuesta tipo Likert y tres alternativas. Los ítems del inventario se distribuyen a partir de dos factores: ansiedad estado y ansiedad rasgo. La consistencia interna para cada subescala se sitúa entre .80 y .90 y la fiabilidad test-retest entre .31 y .71 (Seisdedos, 1990). Con respecto a la consistencia interna se observa un $\alpha$ de Cronbach de .69 para ansiedad estado y .83 para ansiedad rasgo.

Por último, se desarrolló el cuestionario informatizado de normas de circulación (en adelante, test de conducir) que constituía la tarea indirecta de agresión. Dicho instrumento se fundamentaba a partir de los paradigmas de elección de opciones agresivas o no agresivas (Fischer, Guter y Frey, 2008; Fischer et al., 2007; Giumetti y Markey, 2007). El test de conducir contenía 20 ítems con cuatro alternativas de respuesta, desarrollados a partir de los exámenes para la consecución del permiso de conducción, válido en el estado español (Dirección General de Tráfico, DGT, 2012). Cada 
ítem se formaba de dos alternativas de respuesta consideradas agresivas o arriesgadas y otras dos no agresivas; hay que tener en cuenta que ninguna de las alternativas era la respuesta correcta o idónea según las normas de la DGT. Las opciones de respuesta fueron contrabalanceadas con respecto al orden de presentación al participante en las dos sesiones del estudio. Los ítems del cuestionario fueron seleccionados de una lista de posibles propuestas a partir del juicio de dos revisores considerándose la respuesta agresiva aquélla en la que se cometían delitos de circulación o se exponían conductas violentas en la carretera.

En la Figura 1 se muestra la interfaz de presentación del test de conducir. Como medida de la respuesta agresiva se contabilizaba la frecuencia de elección de una de las opciones agresivas para el ítem.

\section{Aparatos}

El experimento se realizó en un aula con 22 ordenadores de sobremesa, Pentium IV ${ }^{\circledR}$, un monitor por cada ordenador, así como teclado, mouse y altavoces (un juego de altavoces por ordenador); y dos ordenadores portátiles, de similar potencia y con los mismos periféricos.

Los videojuegos que se utilizaron fueron: Carmageddon TDR $2000^{\mathbb{R}}$ (Xicat Interactive, 2000), considerado un juego para mayores de 18 años, según la ESA (2012). En dicho organismo este juego se detalla con los siguientes descriptores: animated blood and gore, animated violence (ver también Haninger, Ryan y Thompson, 2004). Se trata de un juego de conducción violenta en el que el protagonista posee un coche y va disputando una carrera en la que tiene que destruir a todos sus rivales así como a los peatones que circulan por las aceras (definidos como zombies en el juego).

Por otro lado, se utilizó el videojuego $3 d$ Driving Schoo ${ }^{\circledR}$ (Besier 3D-Edutainment Wiesbaden, 2001), como condición de juego no violento. Dicho videojuego se trata de un simulador de conducción que pretende servir de entorno virtual de práctica de conducción.

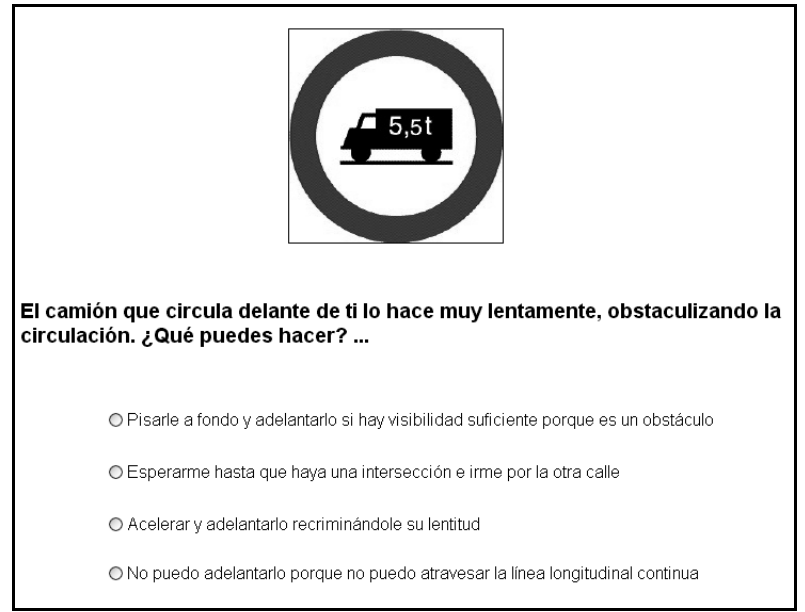

Figura 1. Ejemplo de ítem del test de conducir. Nota.- Las opciones primera y tercera puntuaban con uno.

\section{Procedimiento}

La selección de los participantes se realizó a partir de la entrega a los alumnos, del formulario de consentimiento informado junto con la descripción del estudio, para que fuese revisado y refrendado por los padres o tutores. Pasado un plazo de dos semanas, todos los alumnos que devolvieron el documento cumplimentado por los padres o tutores fueron seleccionados para participar en el estudio.

El desarrollo del estudio se llevó a cabo dividiendo la muestra en dos grupos de 24 y 23 participantes, respectivamente. Ambos grupos pasaron por dos sesiones de exposición a los videojuegos, una sesión para cada tipo de videojuego, con un orden de presentación contrabalanceado (aproximadamente a la mitad de los participantes de cada grupo se exponía al videojuego violento primero y después al no violento; a la otra mitad, se exponía en orden contrario).

Los participantes fueron citados en un aula que contaba con los ordenadores descritos en una sección posterior. A continuación el experimentador les daba instrucciones acerca de que se debía completar previa a la exposición a cada videojuego el STAXI-NA y el STAIC, y cómo se debía hacer. Posteriormente cada participante jugaba a través del teclado y el mouse del ordenador durante 30 minutos al videojuego que le correspondía. Inmediatamente después de la exposición, cada participante debía completar el test de conducir y posteriormente las subescalas de estado del STAXINA y el STAIC.

La segunda sesión se estructuraba con las mismas tareas que la primera salvo que en la fase pretest únicamente se completaban las subescalas de estado del STAXI-NA y el STAIC, en el orden anterior.

\section{Análisis de datos}

Se realizaron análisis de la varianza para determinar el efecto de la exposición a cada tipo de videojuego, edad y sexo; además se llevó a cabo un análisis de un modelo de regresión lineal para corroborar la existencia de factores predictores de la respuesta agresiva tras la exposición al videojuego violento, y al no violento. Para el análisis de los efectos pre-postest se usaron pruebas de comparación de medias $t$ de Student, así como el estadístico $d$ de Cohen para determinar el tamaño del efecto.

Se examinaron los datos procedentes de la edad en función de tres grupos: menores (12 años de edad), medianos (13 años de edad) y mayores (14.31 años de edad promedio).

Además, se analizaron los ítems del test de conducir de forma descriptiva, teniéndose en cuenta las respuestas a los ítems en la primera sesión experimental.

A continuación se extrajeron índices de validez convergente del instrumento, a partir de correlaciones de Pearson con las puntuaciones del STAXI; y fiabilidad, a partir del cálculo del índice alfa de Cronbach para ítems con formato de respuesta dicotómica (Elosua y Zumbo, 2008). Para esto, 
se imputaron los valores perdidos en el test de conducir mediante las iteraciones realizadas con el método de imputaciones múltiples (Acock, 2005).

Todos los análisis se realizaron mediante el paquete estadístico SPSS v. $17.0^{\circledR}$, excepto los análisis de los ítems del test de conducir que se realizaron con el software FACTOR v. 8.02 (Lorenzo-Seva y Ferrando, 2012).

\section{Resultados}

No se encontraron diferencias significativas entre los participantes con respecto al orden de exposición a las diferentes condiciones de la variable tipo de videojuego, momento temporal de la misma o grupo de exposición; asimismo, no se hallaron diferencias significativas con respecto a sexo ni grupo de edad, ni al orden de presentación de los ítems en el test de conducir.

\section{Efectos de la exposición a los videojuegos}

En la Tabla 1 se adjuntan las puntuaciones promedio de los participantes en la tarea del test de conducir y en las pruebas de autoinforme, en función de la exposición a uno u otro tipo de videojuego.

Tabla 1. Puntuaciones de los participantes en función del tipo de videojuego.

\begin{tabular}{llll} 
& & \multicolumn{2}{c}{ Tipo de videojuego } \\
\cline { 3 - 4 } & $N$ & Violento & No violento \\
\hline Test de conducir & 43 & $7.52(3.87)$ & $6.27(3.31)$ \\
Pretest & & & \\
Postest & 38 & $81.17(14.88)$ & $83.45(8.17)$ \\
STAXI-E & 38 & $83.51(8.35)$ & $85.63(8.15)$ \\
Pretest & & & \\
Postest & 44 & $0.21(0.56)^{\mathrm{a}}$ & $0.20(0.55)$ \\
\hline Nota & 40 & $0.42(0.81)^{\mathrm{a}}$ & $0.25(0.97)$
\end{tabular}

Nota.- Los datos indicados en la tabla reflejan la puntuación promedio (con desviación típica). Las puntuaciones del STAIC-E se representan en percentiles.

${ }^{a}$ Diferencias estadísticamente significativas, $\operatorname{con} p<.04$.

El ANOVA que se desarrolló puso de manifiesto la ausencia de un efecto principal del tipo de videojuego en la prueba, pero destacó la presencia de un efecto principal de la edad, con una $F(3,35)=4.01 ; p<.02$ (potencia observada $=.79 ; \eta^{2}$ parcial $\left.=.25\right)$; por otro lado, se encontró un efecto de la interacción entre la edad, sexo y el tipo de videojuego, con una $F(1,35)=242.20 ; p<.01$ (potencia observada $=1$; $\left.\eta_{\text {parcial }}^{2}=.87\right)$.

La prueba post hoc de Bonferroni destacó la presencia de diferencias significativas entre los participantes según los grupos de edad (con $p<.04$ ). En la Figura 2 se representan las puntuaciones de los participantes en el test de conducir en función del sexo y la edad.

En referencia a las puntuaciones del STAXI-E, el ANOVA destacó la ausencia de efectos principales de las variables tipo de videojuego, edad y sexo; pero sí se encontró un efecto de interacción entre las mismas, con $F(1,32)=$ 9.57, $p<.01$ (potencia observada $=.85 ; \eta_{\text {parcial }}^{2}=.23$ ). Los hombres medianos mostraron las puntuaciones más elevadas en el test de conducir, seguidas de las mujeres mayores. Las puntuaciones más bajas se encontraron en los grupos de mujeres de menor y mediana edad. Por otro lado, se observó un efecto pre-postest tras la exposición al videojuego violento, con una $t(23)=3.81 ; p<.04(d=0.33)$.

Por otra parte, cabe señalar que no se encontraron efectos principales de las variables STAXI-R y STAIC-R, ni diferencias significativas en el test de conducir entre los participantes con altos o bajos niveles en dichos factores.

En el caso del STAIC-E, se reveló un efecto de interacción entre el tipo de videojuegos, la edad y el sexo; con $F(2$, $30)=3.54 ; p<.05\left(\right.$ potencia observada $\left.=.61 ; \eta_{\text {parcial }}^{2}=.19\right)$. No se encontraron efectos pre-postest como consecuencia de la exposición a los diferentes niveles de la variable tipos de videojuego.

\section{Análisis del cuestionario informatizado}

En la Tabla 2 se muestra el análisis de los ítems del test de conducir aplicado a los participantes. Así, se presentan datos de promedio y dispersión, además de los índices de frabilidad y validez convergente (correlación con el STAXI Estado).

Tabla 2. Puntuaciones derivadas del análisis de los ítems del test informatizado y la validez.

\begin{tabular}{llll}
\hline & $m(d t)$ & $\alpha$ & $r^{*}$ \\
\hline Test de conducir & $.33(.41)$ & $.86^{*}$ & $.39 * * *$ \\
\hline
\end{tabular}

Notas.- "Correlación bivariada del test de conducir con STAXI Estado tras la exposición al videojuego.

$* * p<.05 ; * * * p<.01$.

Los índices psicométricos del instrumento se muestran adecuados, con una buena fiabilidad como consistencia interna, y una aceptable correlación con una variable asociada a conducta agresiva, como es ira (del Barrio et al., 2004).

\section{Modelos predictores de la exposición a los videojuegos}

Únicamente se mostraron significativas dos ecuaciones de regresión para la condición de videojuego violento. Se escogió el modelo presentado con $F(2,32)=8.60 ; p<.01\left(R^{2}\right.$ ajustada $=.32$, que incluía como predictores la edad y los niveles pretest en el STAXI estado.

Se presenta a continuación el modelo predictor estandarizado de las puntuaciones en la tarea informatizada tras la exposición al videojuego violento.

Puntuación tras TDR $=0.41(\mathrm{Edad})+0.37\left(\mathrm{STAXI}-\mathrm{E}_{\text {pretest }}\right)$

Ningún modelo de regresión lineal se encontró significativo en la condición de videojuego no violento. 


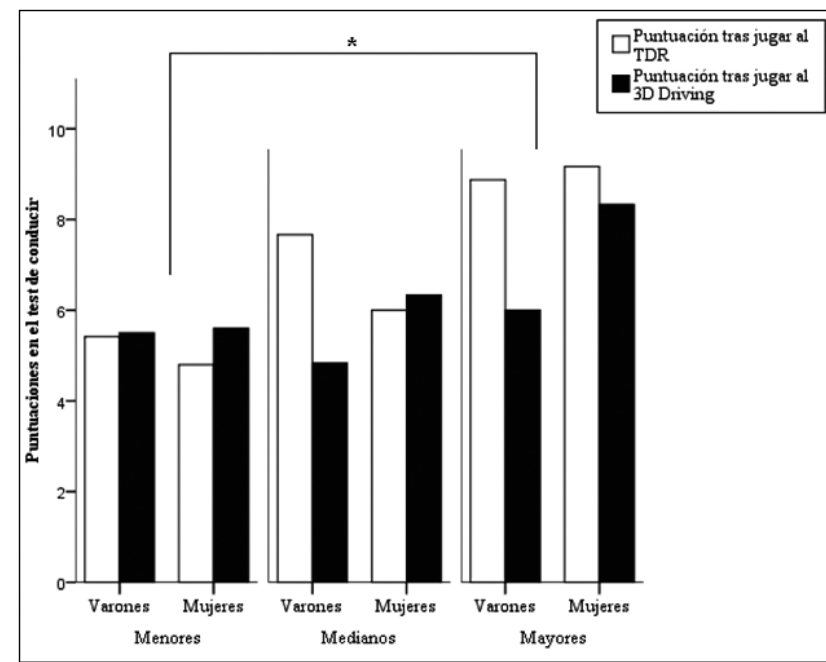

Figura 2. Puntuaciones de los participantes en el test de conducir en función de la edad y el sexo tras la exposición a cada tipo de videojuego.

Nota.- Las puntuaciones en el eje horizontal se distribuyen por grupos de edad: menores $(n=17)$, medianos $(n=12)$ y mayores $(n=14)$. ${ }^{*} p<.05$.

\section{Discusión}

Muchas familias se quejan del excesivo tiempo que sus hijos pasan expuestos a videojuegos violentos y preguntándose cuáles pueden ser los efectos de dicha exposición y las consecuencias en su salud emocional. Sin embargo, se presenta una importante permisividad con respecto a la exposición a contenido violento tanto en videojuegos como en otros soportes media (Álvarez-García et al., 2011; Brady, 2007; Fischer et al., 2011; Huesmann y Taylor, 2006; Ybarra et al., 2008).

Los objetivos del estudio eran evidenciar si la exposición a videojuegos violentos de coches, durante un tiempo limitado, presenta efectos observables en una tarea de agresión indirecta, y en los niveles de ira y ansiedad tras la exposición; y examinar la existencia de variables moduladoras de la respuesta de agresión en el test de conducir y los niveles de ira y ansiedad postest, tales como la edad o el sexo; así como delimitar un modelo predictor para las puntuaciones en dicha tarea.

En la investigación se pone de manifiesto la potencia explicativa de la exposición a los videojuegos violentos en la conducta humana, concretamente en la respuesta agresiva, medida a través de una prueba indirecta. La exposición a videojuegos per se no se presenta como el factor principal que modula la respuesta en la tarea de agresión. La edad se muestra como un factor importante en la respuesta agresiva, así como la interacción del tipo de videojuego con la edad y el sexo.

Además, se señala la presencia de un aumento significativo de la ira tras la exposición al videojuego violento, medida mediante la prueba de autoinforme. Por otro lado, hay que tener en cuenta que la respuesta en la tarea de agresión se puede predecir con un modelo de regresión lineal cuyos factores son la edad y la ira previa a la exposición.

En primer lugar, cabe destacar que en el estudio no se encontraron diferencias significativas en la prueba de agresión indirecta tras la exposición a uno u otro tipo de videojuego. Este dato señala la ausencia de un efecto principal de la exposición a un videojuego de contenido violento sobre la respuesta en la tarea de agresión tras la misma. También otros estudios han encontrado la ausencia de una relación directa entre exposición a videojuegos violentos y respuesta agresiva a corto y largo plazo (Brady, 2007; Ferguson, 2011; Wei, 2007; Yagci y Çaglar, 2009).

Ferguson et al. (2008) realizaron una serie de dos estudios para determinar la asociación de la exposición a los videojuegos violentos con la ejecución de actos delictivos en una muestra de 428 estudiantes universitarios. El modelo de ecuaciones estructurales que se derivó del estudio destacó la ausencia de efecto de la exposición a videojuegos violentos en la práctica de actos delictivos (sí formaron parte del modelo la violencia vista en la familia, el género masculino y la agresión rasgo). Cabe destacar por otro lado, el efecto del videojuego no agresivo, que tras una breve demora, puede provocar un aumento de la respuesta agresiva (Sestir y Bartholow, 2010); produciéndose un decremento de las diferencias entre las condiciones experimentales.

Entre los estudios que sí encontraron un efecto principal del contenido violento sobre la respuesta agresiva (Anderson y Carnagey, 2009; Arriaga et al., 2006; Beullens, Roe y van den Bulck, 2008; Fischer et al., 2008), se plantea la existencia de las variables moduladoras de tal efecto (Anderson et al., 2010). En el presente estudio se encontró un efecto modulador de la edad y el sexo en las puntuaciones en el test tras exponerse al videojuego violento. Otros autores observaron la influencia de variables de personalidad (Barlett et al., 2007; Giumetti y Markey, 2007), sociales (Barlett et al., 2009; Ferguson et al., 2008; Huesmann y Taylor, 2006) y ontogenéticos (Anderson, 2004; Anderson et al., 2010; Bushman y Huesmann, 2006; Wallenius y Punamäki, 2008).

La edad se presenta como una variable con un efecto determinante en la relación videojuegos violentos y respuesta agresiva. En el presente estudio se demuestra que los participantes mayores reflejaban puntuaciones más agresivas que los menores; es decir, a mayor edad mayor puntuación en la tarea de agresión. Asimismo, se indica la presencia de la edad como factor en el modelo predictor de la respuesta en la tarea de agresión tras la exposición al videojuego violento.

La literatura científica ha aportado evidencias a favor de la adolescencia como un periodo con significativamente mayor frecuencia de conductas de tipo agresivo que etapas anteriores del desarrollo (Anderson, 2004; Kirsh, 2003; Wallenius y Punamäki, 2008). Loeber y Stouthamer-Loeber (1998) encontraron que la respuesta agresiva va aumentando desde la infancia hasta alcanzar un pico entre los 13-15 años, disminuyendo a finales de la adolescencia.

Por tanto, la edad parece mostrarse como una variable que modula el priming de la exposición a un videojuego vio- 
lento en la conducta agresiva humana. Si bien, dicha variable interacciona con el tipo de videojuego y el sexo: los participantes de mayor edad mostraban las puntuaciones más altas en la tarea de agresión tras la exposición al videojuego violento, concretamente las mujeres mayores; los participantes varones mostraban mayores puntuaciones que las mujeres en la tarea a excepción del grupo de mayor edad.

Existen diversos estudios que señalan efectos diferenciales en la respuesta agresiva tras la exposición a videojuegos y contenido media violentos, que incluyen efectos moduladores o mediadores de la edad y el sexo conjuntamente (Bluemke et al., 2010; Bushman y Huesmann, 2006; Ferguson y Garza, 2011; Möller y Krahé, 2009; Olson et al., 2007; Wallenius y Punamäki, 2008).

En siguiente lugar, cabe destacar que se encontraron diferencias significativas pre-postest de la ira autoinformada tras la exposición al videojuego violento de coches: los participantes manifestaron niveles de ira mayores que en la preexposición. La exposición a contenido multimedia violento tiene efectos en la ira (Adachi y Willoughby, 2011; Giumetti y Markey, 2007; Wittman et al., 2008). Además, en diversos estudios se ha presentado apoyo empírico a favor de la facilitación de la exposición a contenido de tipo media en la activación de la ira a corto plazo (Huesman, 2007; Hummer et al., 2010; Krahé et al., 2011). Asimismo, algunos autores han señalado los efectos de facilitación debidos a la presentación de contenido violento, tras exposiciones repetidas (Huesmann y Tailor, 2006). Por otro lado, existen evidencias que han relacionado la ira con ruptura de normas de circulación (Gómez-Fraguela y González-Iglesias, 2010).

Con respecto a la ansiedad postest informada por los participantes, se encontró un efecto de interacción entre el tipo de videojuegos, la edad y el sexo. Se observaron las mayores puntuaciones en el STAIC-E en los participantes varón de mayor edad. Existen otros estudios empíricos que muestran efectos de la edad y/o el sexo en la relación entre la exposición a videojuegos violentos y la respuesta ansiogénica (Arriaga et al., 2006; Baldaro et al., 2004; Bluemke et al., 2010).

No se observaron, sin embargo, resultados en la presente investigación a favor de la influencia en la exposición a videojuegos violentos con respecto a la respuesta agresiva.

\section{Referencias}

Acock, A. C. (2005). Working with missing values. Journal of Marriage and Family, 67, 1012-1028.

Adachi, P. J. C. y Willoughby, T. (2010). The effect of violent video games on aggression: Is it more than just the violence? Aggression and Violent Behavior, 16, 55-62.

Agencia EFE. (15 de enero de 2001). El presunto 'asesino de la catana' queda en libertad en Murcia a la espera de juicio. El País. Recuperado día 19 de enero de 2012, de http://www.elpais.com/articulo/ espana/presuto/asesino/catana/queda/libertad/Murcia/espera/juicio/elpe piesp/20010115elpepinac_20/Tes

Álvarez-García, D., Núñez, J. C., Álvarez, L., Dobarro, A., Rodríguez, C. y González-Castro, P. (2011). Violencia a través de las tecnologías de la
El resultado más significativo del estudio tiene que ver con la adecuación de un modelo lineal predictor de la respuesta a la tarea de agresión tras la exposición al videojuego violento. Se encontraron como factores de dicho modelo la edad y la ira estado previa a la exposición. El efecto de la edad se manifiesta modulando las puntuaciones de los participantes. La otra variable predictora que en el estudio se mostró significativa fue la ira estado preexposición. Se observó que los niveles de dicha variable influían en las puntuaciones del test de conducir tras el videojuego violento. Este resultado apoya la hipótesis del efecto de priming de la ira, dado que dicho estado emocional facilita la expresión de la respuesta agresiva a corto plazo (Anderson y Bushman, 2002; Baldaro et al., 2004; Fleming y Rickwood, 2001; Giumetti y Markey, 2007; Huesmann y Taylor, 2006).

A modo de conclusión, de la presente investigación se destaca que la influencia de la exposición a videojuegos violentos produce un efecto significativo en la respuesta agresiva de adolescentes, que se ve modulado por la edad y el sexo. Asimismo, la ira autoinformada también se ve modulada por la exposición a videojuegos violentos de coches de manera transitoria. El estudio presenta evidencias a favor de la capacidad de la ira y la edad como facilitadores, a corto plazo, de la respuesta agresiva tras la exposición al videojuego violento de coches.

Como limitaciones del estudio, señalar la ausencia de medidas psicofisiológicas o de conducta agresiva directa que podrían esclarecer en mayor medida los resultados que se obtuvieron. Asimismo, sería necesario comprobar hasta qué punto todos los videojuegos de coches producen activación, ira u otras emociones dado el grado de competitividad que algunos de éstos presentan.

Se ha generado un importante debate social sobre la influencia de los videojuegos violentos en las respuestas agresivas. Estudios como éste aportan evidencias que permiten concretar el verdadero papel de estas exposiciones, y añaden argumentos a padres y tutores para decidir si realmente desean que sus adolescentes allegados sigan presenciando las escenas de violencia de este tipo de videojuegos. Las líneas de investigación futuras se deben centrar en la determinación de herramientas adecuadas que posibiliten que este tipo de videojuegos conserve su carácter lúdico, pero no su carácter violento.

información y la comunicación en estudiantes de secundaria. Anales de Psicología, 27, 221-231.

Anderson, C. A. (2004). An update on the effects of playing violent video games. Journal of Adolescence, 27, 113-122.

Anderson, C. A. y Bushman, B. J. (2002). Human Aggression. Annual Review of Psychology, 53, 27-51.

Anderson, C. A. y Carnagey, N. L. (2009). Causal effects of violent sports video games on aggression: Is it competitiveness or violent content? Journal of Experimental Social Psychology, 45, 731-739.

Anderson, C. A. y Dill, K. E. (2000). Video games and aggressive thoughts, feelings, and behavior in the laboratory and in life. Journal of Personality and Social Psychology, 78, 772-790. 
Anderson, C. A., Shibuya, A., Ihori, N., Swing, E. L., Bushman, B. J., Sakamoto, A. y Saleen, M. (2010). Violent video game effects on aggression, empathy, and prosocial behavior in eastern and western countries: A meta-analytic review. Psychological Bulletin, 136, 151-173.

Arriaga, P., Esteves, F., Carneiro, P. y Monteiro, M. B. (2006). Violent computer games and their effects on state hostility and physiological arousal. Aggressive Behavior, 32, 146-158.

Baldaro, B., Tuozzi, G., Codispoti, M., Montebarocci, O., Barbagli, F., Trombini, E. y Rossi, N. (2004). Aggressive and non-violent videogames: Short-term psychological and cardiovascular effects on habitual players. Stress and Health, 20, 203-208.

Barlett, C. P., Anderson, C. A. y Swing, E. L. (2009). Video game effects Confirmed, suspected, and speculative. A review of the evidence. Simulation \& Gaming, 40, 377-403.

Barlett, C. P., Harris, R. J. y Bruey, C. (2007). The effect of the amount of blood in a violent video game on aggression, hostility, and arousal. Journal of Experimental Social Psychology, 44, 539-546.

Bartholow, B. D., Sestir, M. A. y Davis, E. B. (2005). Correlates and consequences of exposure to video game violence: Hostile personality, empathy, and aggressive behavior. Personality and Social Psychology Bulletin, 31 , 1573-1586.

Bercedo, A., Redondo, C., Pelayo, R., Gómez, Z., Hernández, M. y Cadenas, N. (2005). Consumo de los medios de comunicación en la adolescencia. Anales de Pediatría, 63, 516-525.

Berkowitz, L. (1984). Some effects of thoughts on anti- and prosocial influence of media events: A cognitive neoassociationist analysis. Psychological Bulletin, 95, 410-427.

Beullens, K., Roe, K. y van den Bulck, J. (2008). Video games and adolescents' intentions to take risks in traffic. Journal of Adolescent Health, 43, 87-90.

Bluemke, M., Friedrich, M. y Zumbach, J. (2010). The influence of violent and nonviolent computer games on implicit measures of aggressiveness. Aggressive Behavior, 36, 1-13

Bösche, W. (2010). Violent video games prime both aggressive and positive cognitions. Journal of Media Psychology, 22, 139-146.

Brady, S. S. (2007). Young adults' media use and attitudes toward interpersonal and institutional forms of aggression. Aggressive Behavior, 33, 519525 .

Bushman, B. J. y Huesmann, R. (2006). Short-term and long-term effects of violent media on aggression in children and adults. Archives of Pediatrics \& Adolescent Medicine, 160, 348-352

Chóliz , M. y Marco, C. (2011). Patrón de uso y dependencia de videojuegos en infancia y adolescencia. Anales de Psicología, 27, 418-426.

Del Barrio, V., Aluja, A. y Spielberger, C. (2004). Anger assessment with the STAXI-CA: Psychometric properties of a new instrument for children and adolescents. Personality and Individual Differences, 37, 227-244.

Del Barrio, V., Aluja, A. y Spielberger, C. (2005). STAXI-NA. Inventario de Expresión de Ira Estado-Rasgo en Niños y Adolescentes. Madrid: TEA Ediciones S. A.

Dirección General de Tráfico. (2012). Test de examen de la DGT. Recuperado el día 19 de enero de 2012, de http://www.dgt.es/ revista/test/index.html

Elosua, P. y Zumbo, B. D. (2008). Coeficientes de fiabilidad para escalas de respuesta categórica ordenada. Psicothema, 20, 896-901.

Entertainment Software Association. (2012). Entertainment Software Rating Board. Recuperado el día 19 de enero de 2012, de http://www.esrb.org/index-js.jsp

Ferguson, C. J. (2011). Video games and youth violence: A prospective analysis in adolescents. Journal of Youth and Adolescence, 40, 377-391.

Ferguson, C. J. y Garza, A. (2011). Call of (civic) duty: Action games and civic behavior in a large sample of youth. Computer in Human Behavior, 27, 770-775.

Ferguson, C. J., Rueda, S. M., Cruz, A. M., Ferguson, D. E., Fritz, S. y Smith, S. M. (2008). Violent video games and aggression: Causal relationship or byproduct of family violence and intrinsic violence motivation? Criminal Justice and Behavior, 35, 311-332.

Fernández, J. y Noblejas, M. (2007). Cómo informar sobre infancia y violencia. Valencia: Centro Reina Sofía.

Fischer, P., Greitemeyer, T., Kastenmüller, A., Vogrincic, C. y Sauer, A. (2011). The effects of risk-glorifying media exposure on risk-positive cognitions, emotions, and behaviors: A meta-analytic review. Psychological Bulletin, 137, 367-390.

Fischer, P., Guter, S. y Frey, D. (2008). The Effects of risk-promoting media on inclinations toward risk-taking. Basic and Applied Social Psychology, 30, 230-240.

Fischer, P., Kastenmüller, A. y Greitemeyer, T. (2010). Media violence and the self: The impact of personalized gaming characters in aggressive video games on aggressive behavior. Journal of Experimental Social Psychology, 46, 192-195.

Fischer, P., Kubitzki, J., Guter, S. y Frey, D. (2007). Virtual driving and risk taking: Do racing games increase risk-taking cognitions, affect, and behaviors? Journal of Experimental Psychology: Applied, 13, 22-31.

Fleming, M. J. y Rickwood, D. J. (2001). Effects of violent versus nonviolent video games on children's arousal, aggressive mood, and positive mood. Journal of Applied Social Psychology, 31, 2047-2071.

Gentile, D. A. (2009). Pathological video-game use among youth ages 8 to 18: A national study: Research article. Psychological Science, 20, 594-602.

Gentile, D. A. y Walsh, D. A. (2002). A normative study of family media habits. Journal of Applied Developmental Psychology, 23, 157-178.

Giumetti, G. W. y Markey, P. M. (2007). Violent video games and anger as predictors of aggression. Journal of Research in Personality, 41, 1234-1243.

Gómez-Fraguela, J. A. y González-Iglesias, B. (2010). El papel de la personalidad y la ira en la explicación de las conductas de riesgo al volante en mujeres jóvenes. Anales de Psicología, 26, 318-324.

Hallal, P. C., Bertoldi, A. D., Gonçalves, H. y Victora, C. G. (2006). Prevalência de sedentarismo e fatores associados em adolescentes de 10-12 anos de idade (Prevalencia del sedentarismo y sus factores asociados en adolescentes de 10-12 años de edad). Cadernos de Saúde Pública, 22, $1277-$ 1287.

Haninger, K., Ryan, M. S. y Thompson, K. M. (2004). Violence in teen-rated video games. Medscape General Medicine, 6.

Higuchi, S., Motohashi, Y., Liu, Y. y Maeda, A. (2005). Effects of playing a computer game using a bright display on presleep physiological variables, sleep latency, slow wave sleep and REM sleep. Journal of Sleep Research, 14, 267-273.

Holtz, P. y Appel, M. (2011). Internet use and video gaming predict problem behavior in early adolescence. Journal of Adolescence, 34, 49-58.

Huesmann, L. R. (2007). The impact of electronic media violence: Scientific theory and research. Journal of Adolescent Health, 41, S6-S13.

Huesmann, L. R. y Taylor, L. D. (2006). The role of media violence in violent behavior. Annual Review of Public Health, 27, 393-415.

Hummer, T. A., Wang, Y., Kronenberger, W. G., Mosier, K. M., Kalnin, A. J., Dunn, D. W. y Mathews, V. P. (2010). Short-term violent video game play by adolescents alters prefrontal activity during cognitive inhibition. Media Psychology, 13, 136-154.

Jansz, J. (2005). The emotional appeal of violent video games for adolescent males. Communication Theory, 15, 219-241.

Kerr, M. A. y Schneider, B. H. (2008). Anger expression in children and adolescents: A review of the empirical literature. Clinical Psychology Review, 28, 559-577.

Kirsh, S. J. (2003). The effects of violent video games on adolescents: The overlooked influence of development. Aggression and Violent Behavior, 8, 377-389.

Krahé, B., Möller, I., Huesmann, L. R., Kirwil, L., Felber, J. y Berger, A. (2011). Desensitization to media violence: Links with habitual media violence exposure, aggressive cognitions, and aggressive behavior. Journal of Personality and Social Psychology, 100, 630-646.

Loeber, R. y Stouthamer-Loeber, M. (1998). Development of juvenile aggression and violence: Some common misconceptions and controversies. American Psychologist, 53, 242-259.

Lorenzo-Seva, U. y Ferrando, P. J. (2012). FACTOR. Recuperado el día 19 de enero de 2012, dehttp://psico.fcep.urv.es/utilitats/factor/ index.html

Mathiak, K. y Weber, R. (2006). Toward brain correlates of natural behavior: fMRI during violent video games. Human Brain Mapping, 27, 948-956.

Möller, I. y Krahé, B. (2009). Exposure to violent video games and aggression in German adolescents: A longitudinal analysis. Aggressive Behavior, 35, 75-89. 
Olds, T., Wake, M., Patton, G., Ridley, K., Waters, E., Williams, J. y Hesketh, K. (2009). How do school-day activity patterns differ with age and gender across adolescence? Journal of Adolescent Health, 44, 64-72.

Olson, C. K., Kutner, L. A., Warner, D. E., Almerigi, J. B., Baer, L., Nicholi, A. M., II, y Beresin, E. V. (2007). Factors correlated with violent video game use by adolescent boys and girls. Journal of Adolescent Health, 41, 77-83.

Panee, C. D. y Ballard, M. E. (2002). High vs. low aggressive priming during video-game training: Effects on violent action during game play, hostility, heart rate, and blood pressure. Journal of Applied Social Psychology, 32, 2458-2474.

Salminen, M. y Ravaja, N. (2008). Increased oscillatory theta activation evoked by violent digital game events. Neuroscience Letters, 435, 69-72.

Seisdedos, N. (1990). STAIC: cuestionario de autoevaluación. Madrid: TEA Ediciones S. A.

Sestir, M. A. y Bartholow, B. D. (2010). Violent and nonviolent video games produce opposing effects on aggressive and prosocial outcomes. Journal of Experimental Social Psychology, 46, 934-942.

Sharif, I. y Sargent, J. D. (2006). Association between television, movie, and video game exposure and school performance. Pediatrics, 118, e1061e1071.

Skoric, M. M., Teo, L. L. C. y Neo, R. L. (2009). Children and video games: Addiction, engagement, and scholastic achievement. Cyberpsychology and Behavior, 12, 567-572.
Slater, M. D., Henry, K. L., Swaim, R. C. y Anderson, L. L. (2003). Violent media content and aggressiveness in adolescents: A downward spiral model. Communication Research, 30, 713-736.

Spielberger, C. D. (1973). Manual for the state-trait anxiety inventory for children. Palo Alto, CA: Consulting Psychologists Press.

Wallenius, M. y Punamäki, R. L. (2008). Digital game violence and direct aggression in adolescence: A longitudinal study of the roles of sex, age, and parent-child communication. Journal of Applied Developmental Psychology, 29, 286-294.

Wei, R. (2007). Effects of playing violent videogames on Chinese adolescents' pro-violence attitudes, attitudes toward others, and aggressive behavior. Cyberpsychology \& Behavior, 10, 371-380.

Wittmann, M., Arce, E. y Santisteban, C. (2008). How impulsiveness, trait anger, and extracurricular activities might affect aggression in school children. Personality and Individual Differences, 45, 618-623.

Yagci, E. y Çaglar, M. (2010). How the use of computer types and frequency affects adolescences towards anger and aggression. The Turkish Online Journal of Educational Technology, 9, 89-97.

Ybarra, M. L., Diener-West, M., Markow, D., Leaf, P. J., Hamburger, M. y Boxer, P. (2008). Linkages between internet and other media violence with seriously violent behavior by youth. Pediatrics, 122, 929-937.

(Artículo recibido: 26-06-2011, revisado: 18-01-2012, aceptado: 12-03-2012) 